\title{
Real-World Assessments of mySugr Mobile Health App
}

\author{
Fredrick Debong, ${ }^{1}$ Harald Mayer, MD, MSc, MBA, ${ }^{2}$ and Johanna Kober, $\mathrm{PhD}^{2}$
}

\begin{abstract}
Mobile health (mHealth) solutions such as diabetes self-management apps improve glycated hemoglobin, particularly those that provide a feedback loop between patient and health care provider. mHealth apps that incorporate behaviorally designed interventions can improve patient access to diabetes self-management education and ongoing support. The mySugr mobile app was designed to support patients in their diabetes self-management. Most studies of mHealth apps were conducted under controlled conditions and did not elucidate the nuances of patient perceptions and utilization of these apps in everyday life. In this article, we discuss findings from real-world observations of changes in glycemic control and patient satisfaction associated with the use of the mySugr mHealth app.
\end{abstract}

Keywords: Diabetes, Smartphone, App, mHealth, $\mathrm{HbA}_{1 \mathrm{c}}$, Coaching.

\section{Introduction}

$\mathbf{L}$ ARGE RANDOMIZED CONTROLLED TRIALS (RCTs) have demonstrated that achieving target glucose levels early in diabetes leads to better microvascular and macrovascular outcomes. ${ }^{1-6}$ However, despite the continuing introduction of new medications and medical device technologies, a significant percentage of individuals with diabetes are not achieving their treatment goals. ${ }^{7-9}$

Suboptimal adherence to prescribed diabetes treatment regimens is often cited as the underlying cause a key of poor glycemic control. ${ }^{10,11}$ Although affordability of medications and health care services and medication side effects remain key obstacles to treatment adherence, other factors such as perceptions of treatment efficacy, poor health literacy, lack of social support, and the daily burden of self-management also impact patients' ability/willingness to follow their treatment regimens. ${ }^{10,12}$

The ongoing responsibility of administering medication, testing their glucose, and adhering to prescribed lifestyle behaviors can be overwhelming for many patients. Moreover, patients often do not document their self-management activities, which make it difficult for patients and their health care providers to identify problems and adjust therapy. Accurate and complete documentation is essential to effective diabetes management. However, when viewing diabetes mostly as a "data-driven" disease, the psychological burden and its impact on individuals are often overlooked.
Although diabetes management software has been available since the early 1980s, the rapid growth in digital communications technologies (e.g., smartphones, tablets) has prompted the development of a plethora of new selfmanagement tools for patients via mobile health (mHealth) apps. In their 2017 report, Izahar et al. ${ }^{13}$ identified 346 mHealth apps specifically for self-diabetes management that were available from the Apple iTunes and/or Google Play app stores.

Meta-analyses and systematic reviews have shown that mHealth solutions such as diabetes self-management apps improve glycated hemoglobin $\left(\mathrm{HbA}_{1 \mathrm{c}}\right)$, particularly those that provide a feedback loop between patient and health care provider. ${ }^{14,15}$ A recent meta-analysis by Grohmann-Izay and Forisch found that digital tracking and remote coaching were associated with a notable $\mathrm{HbA}_{1 \mathrm{c}}$ reduction $(-0.38 \%)$ in adult populations. ${ }^{16}$ As reported by Greenwood et al., mHealth apps that incorporate behaviorally designed interventions can improve patient access to diabetes self-management education and ongoing support. ${ }^{14}$

However, because the majority of studies included in these reports were controlled trials and focused mainly on change in $\mathrm{HbA}_{1 \mathrm{c}}$ as the primary outcome, they do not elucidate the nuances of patient perceptions and utilization of these apps in everyday life-insight into the real-world impact of such devices is lacking. In this article, we discuss findings from realworld observations of changes in glycemic control and patient satisfaction associated with the use of the mHealth app.

\footnotetext{
${ }^{1}$ hi.health, Vienna, Austria.

${ }^{2}$ mySugr GmbH, Vienna Austria.
} 


\section{mySugr mHealth app}

Launched in 2012, the mySugr mobile app was designed to support patients in the diabetes self-management areas recommended by the American Association of Diabetes Educators 7 Healthy Behaviors curriculum: healthy eating, being active, monitoring, taking medication, risk reduction, problem solving, and healthy coping. ${ }^{17}$ The mySugr app is CEmarked (Conformité Européene), classified as a class I medical device in the European Union, the bolus calculator as class IIb has received the CE (Conformité Européene) mark and is registered classified as a class I medical device with the Food and Drug Administration in the United States. It is available in over 60 countries in 14 languages.

Data from self-monitoring of blood glucose (SMBG) and continuous glucose monitoring devices can be uploaded automatically, either directly via Bluetooth or, for instance, Apple Health, and can also be synced between devices via a cloud-based service. Additionally, insulin data can be entered manually into the app. When users want to make a new entry, they can add pictures and other information to document meals, medications, and activities. Other information (e.g., times, locations) and physical activity levels can be imported automatically from other apps, adding context to the clinical data. Users can access and download all their data as CSV, Excel spreadsheets, or PDF report, giving patients, as well as clinicians, an accurate therapy overview that presents statistics and detailed logs.

A critical and recent offering of the mySugr app is the immediate access to certified diabetes educators (CDEs) when needed. Assisted by algorithms that detect problematic glucose patterns, CDEs identify and monitor at-risk patients and reach out to them with individualized glucose-centric coaching/advice via the application. This population management service, together with a connected device and unlimited test strips delivered directly to the door of the user, makes up the mySugr Bundle, which is reimbursed by payers and health systems both in the European Union and in the United States.

The application further utilizes algorithms for pattern detection, indicating areas that a patient should focus on improving. This functionality in pattern recognition was developed in collaboration with over 700 current users, as well as a team of diabetes educators and clinicians.

In the European Union, the application also features a CEmarked bolus calculator to assist users in their insulin dose calculations, also advising on carbohydrate corrections in the case of a predicted low blood glucose.

Unlike other approaches, which focus primarily on diabetes data, the mySugr app provides users with a mix of positive psychology and assistance in their day-to-day diabetes management. The goal was to design an app that is visually pleasing and provides positive feedback to users about their daily self-management successes. The app uses components from behavioral psychology, deployed as gamemechanics, such as challenges and direct positive feedback loops through avatars and, at the same time, covers all the diabetes data and functionalities needed for effective diabetes management. Importantly, the app enables users to learn from past experiences. Users can search their historical data regarding past events (e.g., meals, activities, moods, locations) and assess the impact of those events on glucose levels and other metrics. With this information, users can make more appropriate therapy decisions based on past experiences in similar situations.

\section{Real-World Assessments of Efficacy and User Satisfaction}

While RCTs are generally considered the "gold standard" for medical research, they often fail to reflect actual patient behaviors and resultant outcomes in real-world clinical practice. $^{18,19}$ Because diabetes self-management depends heavily on patient attitudes and behaviors, different research approaches are needed to more definitively assess behaviorbased interventions such as using mHealth apps in real-world settings. To obtain this evidence, retrospective observational studies were conducted to assess the impact of mySugr use on glycemic control and user satisfaction.

\section{Early analyses}

The following is a summary of studies conducted from 2015 through 2016, investigating the impact of the initial mySugr app version, which did not include features such as CDE-led coaching, unlimited blood glucose test strips, and bolus calculation. These features and others were subsequently added in 2017 .

Retrospective analysis of impact on SMBG and glycemic control of mHealth application for diabetes management. In this 2015 retrospective observational study, researchers investigated the potential impact of the mySugr app usage on parameters of blood glucose control. ${ }^{20} \mathrm{~A}$ randomly selected sample of 2104 highly engaged users (logging $\geq 5$ days/week for $\geq 6$ months) were included. Participant characteristics were as follows: type 1 diabetes (T1D), aged $34.5 \pm 16.13$ years, and $45.77 \%$ were female. The group was not filtered for blood glucose control at baseline. No further inclusion criteria regarding mean blood glucose results or estimated $\mathrm{HbA}_{1 \mathrm{c}}$ $\left(\mathrm{eA}_{1 \mathrm{c}}\right)$ was applied for inclusion in the sample, which may have led to an underestimation of the magnitude of changes in metabolic control. Changes in mean blood glucose, standard deviation (SD), and coefficient of variability (CV) within the group were analyzed at baseline $\left(t_{0}\right)$, month $1\left(t_{1}\right)$, and months 2-6 $\left(t_{2}\right)$, using R software for analyses. Baseline blood glucose results $\left(t_{0}\right)$ were $162.10 \pm 59.25 \mathrm{mg} / \mathrm{dL}$, representing a relatively well-controlled group with an $\mathrm{e}_{1 \mathrm{c}}$ of $7.3 \%$. Baseline data were based on an intercept of regression model of all data from $t_{1}$.

At $t_{1}$, the mean blood glucose had dropped significantly and further at $t_{2},(P<0.01)$ with a stable reduction in glycemic variability SD (Fig. 1). The reduction of mean blood glucose between $t_{0}$ and $t_{2}$ corresponds to a reduction of $\mathrm{eA}_{1 \mathrm{c}}$ of $\sim 0.3 \%$ in an already well-controlled population (from $7.3 \%$ to $7.0 \%$ ) using the conventional conversion method. ${ }^{21}$

Significant improvement of blood glucose control in a high-risk population of T1D using an mHealth app: a retrospective observational study. This retrospective observational study investigated the potential impact of the mySugr app usage on glycemic control in 440 randomly selected, high-risk T1D patients (aged 30.8 \pm 15.3 years, $47.3 \%$ female) over a 6-month period. ${ }^{22}$ Inclusion criteria were as follows: high engagement with the app (logging in $\geq 5$ 
days/week for $\geq 6$ months) and mean blood glucose $\geq 183 \mathrm{mg} / \mathrm{dL}$ (equivalent to $\mathrm{A}_{1 \mathrm{c}}>8.0 \%$ ) at baseline. Changes from baseline $\left(t_{0}\right)$ in mean blood glucose, $\mathrm{SD}$, high blood glucose index (HBGI), and low blood glucose index (LBGI) were assessed at weeks 2-4 $\left(t_{1}\right)$ and months 3-6 $\left(t_{2}\right)$.

At 6 months, the mean blood glucose levels had decreased by $17.9 \%$, from $210.8 \pm 69.4 \mathrm{mg} / \mathrm{dL}$ at baseline to $173.08 \pm$ $63.26 \mathrm{mg} / \mathrm{dL}(P<0.0001)$, and a significant $8.79 \%$ reduction in SD was observed $(P<0.005)$. HBGI had dropped from 5.39 at $t_{0}$ to $3.36(P<0.0001)$, from medium to low risk, ${ }^{23}$ whereas LBGI rose from 0.22 to $0.44(P<0.0001){ }^{23}$ The reduction in mean blood glucose at $t_{2}$ corresponds with a $1.3 \%$ reduction in $\mathrm{eA}_{1 \mathrm{c}}$ (from $9 \%$ to $7.7 \%$ ) using the conventional conversion method. ${ }^{21}$

High-risk population using mobile logging application shows significant reduction in LBGI. This analysis looked into the impact of using the mySugr app on hypoglycemia risk as measured by the LBGI. ${ }^{23,24}$ Data from 4000 engaged (logging $\geq 5$ days/week for $\geq 6$ months) T1D users from the active mySugr user-base were anonymized and exported for analysis. Among these, 1837 users with complete and consistent data sets were analyzed. From this group, 457 users (aged 35.51 \pm 15.58 years, 50.3\% male) with the highest quartile regarding LBGI at $t_{0}$ (days $1-3$ ) were selected for analysis. Changes from baseline $\left(t_{1}\right)$ in mean blood glucose, $\mathrm{SD}$, HBGI, and LBGI were assessed at months 4-6 $\left(t_{2}\right)$. Baseline was selected as $t_{1}$ (days 4-7) to mitigate for regression to the mean in the sample, as it was selected based on extreme LBGI values at $t_{0}$. Subanalyses were conducted to determine if the effect of app use was different between users in four countries and if gender impacted LBGI outcomes, hypothesizing that these two factors may affect baseline data and potentially also could affect the outcomes. The within-population shift between LGBI risk levels was also investigated.

At $t_{2}$, the time period of months $4-6$, a reduction in LBGI of $17.39 \%$, from 1.07 to $0.88(P<0.001)$, was observed accompanied by a small but statistically significant increase in mean blood glucose, from 141.27 to $147.82 \mathrm{mg} / \mathrm{dL}$ $(P<0.001)$. Changes in SD, CV, and HBGI were not significant. Reductions in LBGI were not influenced by nationality or gender. A consistent shift of patients to lower risk levels was also observed (Fig. 2).

\section{Recent analyses}

Clinically relevant improvement in quality of blood glucose control in well-controlled users of the mySugr mobile diabetes management tool. This retrospective study explored real-world changes in blood glucose parameters in a U.S. randomly selected population of mySugr "Bundle" users in the United States. ${ }^{25}$ The mySugr Bundle offers unlimited test strip delivery and CDE-led coaching on top of the normal features of the mySugr app.

Study participants included 52 app users; $55.8 \%$ with T1D, $36.5 \%$ with type 2 diabetes (T2D), $5.8 \%$ with latent autoimmune diabetes in adults (LADA), and three with unreported diabetes type. Of these, $77.1 \%$ used insulin, $19.4 \%$ used insulin pumps, and $22.9 \%$ used noninsulin therapies. Baseline blood glucose was $154.3 \pm 55.5 \mathrm{mg} / \mathrm{dL}$. Median $\mathrm{eA}_{1 \mathrm{c}}$ at baseline was $6.7 \%$.

Measured outcomes included the following: changes in mean blood glucose, $\mathrm{SD}, \mathrm{CV}$, e $\mathrm{A}_{1 \mathrm{c}}$, number of blood glucose tests in range, and frequency of blood glucose testing. Data from the first 2 weeks of use $\left(t_{0}\right), 2$ months before $\left(t_{1}\right)$, and 2 months after $\left(t_{2}\right)$ initiation of mySugr Bundle usage were aggregated and statistically compared using two-sided $t$-tests. A subgroup analysis was also performed, splitting the group by the median $\mathrm{eA}_{1 \mathrm{c}}$ at baseline $\left(t_{0}\right)$.

Significant improvements were observed in the mean blood glucose $(-10.4 \%)$, tests in range $(+8.5 \%)$, and $\mathrm{eA}_{1 \mathrm{c}}$ $(-0.4 \%)$ between $t_{0}$ and $t_{2}$ (Table 1$)$. Significant improvement was also observed in monitoring frequency $(+17.51 \%)$ at $t_{2}$ versus $t_{1}$. A clinically relevant change in $\mathrm{eA}_{1 \mathrm{c}}(\geq 0.3 \%)^{26}$ was achieved by $30.8 \%$ of the population. Subgroup analysis showed greater improvements in $\mathrm{eA}_{1 \mathrm{c}}(-1.1 \%, P<0.001)$, mean blood glucose $(-39.3 \mathrm{mg} / \mathrm{dL}, P<0.001)$, and glycemic variability $(\mathrm{SD})(-14.06 \mathrm{mg} / \mathrm{dL}, P=0.007)$ among participants with baseline e $A_{1 c} \geq 6.7 \%$. No significant changes were observed in the subgroup of participants with baseline $\mathrm{eA}_{1 \mathrm{c}}$ $<6.7 \%$.

Strong customer satisfaction among users of mobile diabetes management. In this study, customer satisfaction among a U.S.-based population with T2D who received at least one shipment of test strips was sent a satisfaction survey (Net Promoter Survey [NPS]) via e-mail. ${ }^{27}$ NPS is a recognized survey tool that assesses satisfaction on a scale of 1-10 based on customer responses to one key question: How likely is it that you would recommend [product/brand] to a friend or

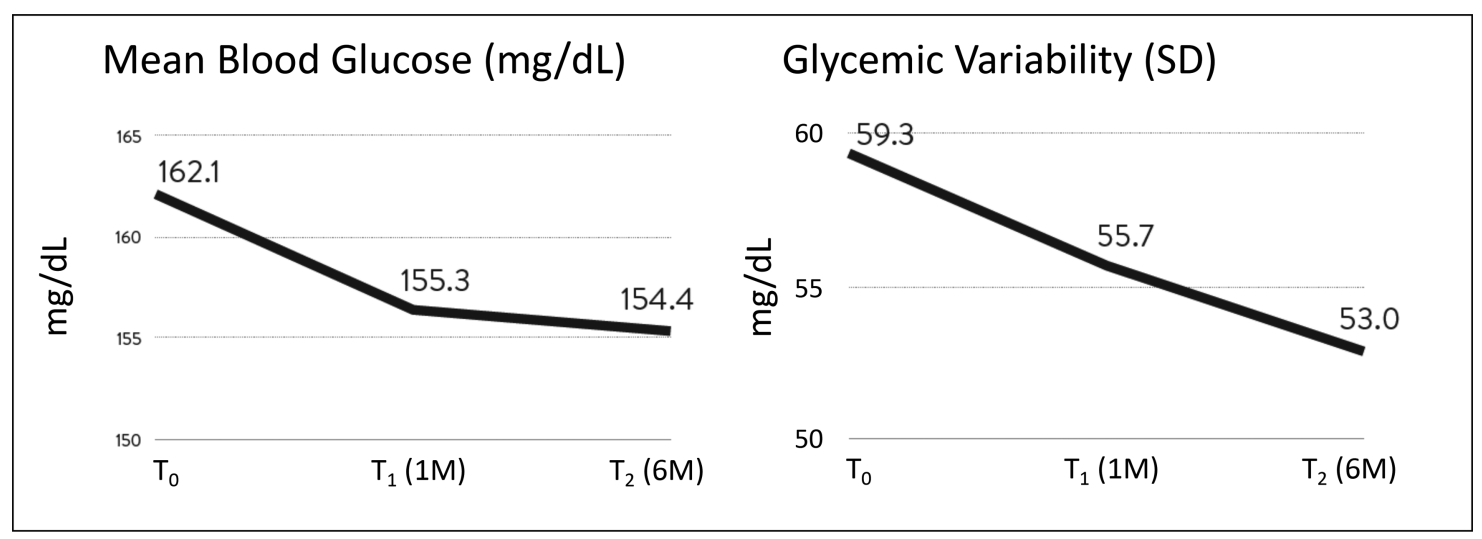

FIG. 1. Change in mean blood glucose and glycemic variability (SD) from $t_{0}$ to $t_{2}$. SD, standard deviation. 


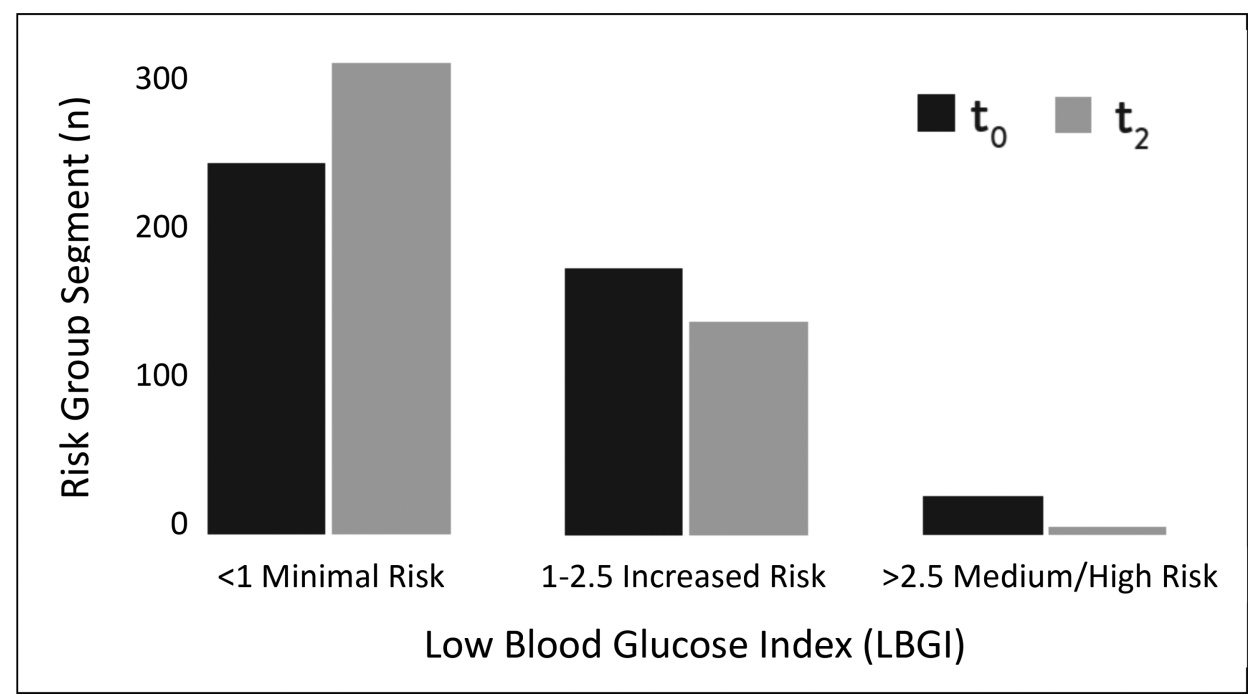

FIG. 2. Shift in size of population groups segmented by hypoglycemia risk levels between $t_{0}$ and $t_{2}$.

colleague $?^{28,29}$ Respondents are grouped into three categories: promoters (score 9-10) are loyal enthusiasts; passives (score 7-8) are satisfied but unenthusiastic; and detractors (score 0-6) are unhappy users. The NPS is calculated by subtracting the percentage of detractors from the percentage of promoters. Scores can range from -100 (100\% detractors) to 100 (100\% promoters).

The survey was sent out in May 2018 and resulted in 105 replies. Approximately $54.3 \%$ of respondents had lived with diabetes for more than 5 years and $45.7 \%$ measured their blood glucose three to five times a day.

The results showed 9 detractors, 80 promoters, and 13 passives, resulting in an NPS of 70, which is comparable to NPS ratings for Netflix (68) and Apple (72). Among respondents who had communicated with a mySugr CDE via smartphone, $86 \%$ reported that they were satisfied with the coaching service.

\section{Discussion}

Patient perceptions of treatment efficacy, deficits in diabetes self-management skills and knowledge, lack of social support, and the daily burden of managing their disease are negatively impacting patient adherence to prescribed treatment regimens. ${ }^{10,12}$ Diabetes self-management education and support has been shown to improve glycemic control, reduce the risk of developing debilitating and life-threatening complications, and positively affect many of the psychoso- cial and behavioral aspects of diabetes. ${ }^{30}$ However, many patients do not have ready access to diabetes education programs. ${ }^{31}$ Given the growing global diabetes epidemic and rising costs associated with its long-term complications, there is an immediate need for new approaches that assist and engage patients in their self-management efforts in ways that support treatment adherence and lessen the burden of diabetes.

One way of supporting patients in their daily selfmanagement is through the use of mHealth apps, which are emerging as a promising solution for a growing number of individuals with diabetes. ${ }^{14-16,32,33}$ Although findings from RCTs have provided strong evidence supporting the use of mHealth apps, relevant and sufficient real-world data that would convincingly demonstrate the usefulness of mHealth tools in clinical care or less controlled settings are lacking, as many mHealth projects have never gone beyond pilot stage.

The mySugr app is the most widely used mobile solution in the field of digital diabetes care, reaching more than 1.5 million individuals with diabetes in over 60 countries. Findings from the early studies suggest that that simply logging with the mySugr app may have positively impacted the quality of blood glucose control. It was hypothesized that the addition of the "Bundle" features (e.g., CDE-led coaching, bolus advisor, and other features), which are included in latest mySugr versions, would have likely resulted in further improvements. This hypothesis appears to have been correct.

Table 1. Changes in Glycemic Measures

\begin{tabular}{lrrrrr}
\hline Value & \multicolumn{1}{c}{$\mathrm{t}_{0}$} & \multicolumn{1}{c}{$\mathrm{t}_{1}$} & $\mathrm{t}_{2}$ & P-value, $\mathrm{t}_{0} \rightarrow \mathrm{t}_{1}$ & $\mathrm{P}_{\text {-value, }} \mathrm{t}_{\boldsymbol{l}} \rightarrow \mathrm{t}_{2}$ \\
\hline Mean BG (mg/dL) & 154.3 & 140.4 & 138.2 & $\mathbf{0 . 0 1 1}$ & 0.378 \\
BG SD (mg/dL) & 55.5 & 52.1 & 50.3 & 0.071 & 0.235 \\
eA $(\%)$ & 6.7 & 6.3 & 6.3 & $\mathbf{0 . 0 1 1}$ & 0.378 \\
Tests in range (\%) & 64.5 & 73.5 & 73.0 & $\mathbf{0 . 0 2 1}$ & 0.677 \\
Test frequency (tests/day) & 5.8 & 5.3 & 6.2 & 0.239 & $<\mathbf{0 . 0 0 1}$ \\
\hline
\end{tabular}

The changes experienced by the population were significant (values shown in boldface) in terms of BG control, test frequency, as well as tests in range when comparing following periods with baseline $t_{0}$.

$\mathrm{BG}$, blood glucose; $\mathrm{eA}_{1 \mathrm{c}}$, estimated $\mathrm{HbA}_{1 \mathrm{c}} ; \mathrm{SD}$, standard deviation; $t_{0}$, first 2 weeks of use; $t_{1}, 2$ months before use; $t_{2}, 2$ months after mySugr initiation. 
As shown in the most recent studies, findings suggest that the use of the bundled mySugr app can prompt positive changes in glucose control in a very short time period and that the impact may be even greater in individuals with less wellcontrolled diabetes. Importantly, the positive trend data on mean blood glucose and glycemic variability further suggest that educator-led coaching unlimited access to blood glucose test strips has a positive impact on both user satisfaction and, importantly, the sustainability of favorable changes, which may lead to long-term improvements in glucose control. Interestingly, although the mySugr Bundle was primarily designed with people with T1D in mind, its value and potential for individuals with T2D was also demonstrated.

Although these studies provide valuable insights into realworld use of the mySugr app, the reported findings are limited by their retrospective observational design. As such, the findings reported here are not conclusive nor can they be generalized to other mHealth products or technologies. Nevertheless, many health care providers with product developers and regulatory agencies recognize the inherent limitations of RCTs in providing real-world evidence (RWE) about the impact and efficacy of medical devices in clinical practice, and an increasing number of payers and regulatory agencies are now focusing on RWE in combination with RCT findings to inform their decisions. ${ }^{34}$

The data presented here will be utilized to generate future research hypotheses to further test the clinical utility of mySugr's integrated diabetes management solutions and to improve the mySugr app and diabetes management tools. Additionally, the observations and conclusions will inform the design of future prospective observational and interventional studies.

mHealth tools have tremendous potential in helping patients achieve their self-management goals and improve the quality of diabetes care. However, as mHealth technologies continue to mature, it is important that developers step away from the "one size fits all" approach to product design. It is important that these technologies have the flexibility to address specific problem(s) and personality of each user; different users will require different solutions. Developers will also need to consider the changing regulatory environment in which both user privacy and post-market surveillance are becoming key issues.

Effective diabetes management is essential to achieving positive health outcomes. mHealth apps that address both the clinical and psychological burden of diabetes have the potential to reduce the burden and enhance patient selfmanagement. However, with the combination of positive feedback, useful advice and convenience is critical to the usability, efficacy, and sustained use of these innovative tools.

\section{Acknowledgments}

The authors thank Christopher G. Parkin, CGParkin Communications, Inc. (Henderson, NV) for editorial assistance in developing this article. The authors received no funding for the development of this article.

\section{Author Disclosure Statement}

H.M. and J.K. are employees of mySugr. F.D. was employed by mySugr at the time this article was written.

\section{References}

1. UK Prospective Diabetes Study (UKPDS) Group: Effect of intensive blood-glucose control with metformin on complications in overweight patients with type 2 diabetes (UKPDS 34). Lancet 1998;352:854-865.

2. Holman RR, Paul SK, Bethel MA, et al.: 10-year follow-up of intensive glucose control in type 2 diabetes. N Engl J Med 2008;359:1577-1589.

3. Diabetes Control and Complications Trial Research Group, Nathan DM, Genuth S, et al.: The effect of intensive treatment of diabetes on the development and progression of long-term complications in insulin-dependent diabetes mellitus. N Engl J Med 1993;329:977-986.

4. Ismail-Beigi F, Craven T, Banerji MA, et al.: Effect of intensive treatment of hyperglycaemia on microvascular outcomes in type 2 diabetes: an analysis of the ACCORD randomised trial. Lancet 2010;376:419-430.

5. ADVANCE Collaborative Group, Patel A, MacMahon S, et al.: Intensive blood glucose control and vascular outcomes in patients with type 2 diabetes. N Engl J Med 2008; 358:2560-2572.

6. Hayward RA, Reaven PD, Wiitala WL, et al.: Follow-up of glycemic control and cardiovascular outcomes in type 2 diabetes. N Engl J Med 2015;372:2197-2206.

7. Carls G, Huynh J, Tuttle E, et al.: Achievement of glycated hemoglobin goals in the US remains unchanged through 2014. Diabetes Ther 2017;8:863-873.

8. Khunti K, Gomes MB, Pocock S, et al.: Therapeutic inertia in the treatment of hyperglycaemia in patients with type 2 diabetes: a systematic review. Diabetes Obes Metab 2018; 20:427-437.

9. Stone MA, Charpentier G, Doggen K, et al.: Quality of care of people with type 2 diabetes in eight European countries: findings from the Guideline Adherence to Enhance Care (GUIDANCE) study. Diabetes Care 2013;36:2628-2638.

10. Garcia-Perez LE, Alvarez M, Dilla T, et al.: Adherence to therapies in patients with type 2 diabetes. Diabetes Ther 2013;4:175-194.

11. Polonsky WH, Henry RR: Poor medication adherence in type 2 diabetes: recognizing the scope of the problem and its key contributors. Patient Prefer Adherence 2016;10:1299-1307.

12. Ross SA: Breaking down patient and physician barriers to optimize glycemic conrol in type 2 diabetes. Am J Med 2013;126(Suppl. 1):S38-S48.

13. Izahar S, Lean QY, Hameed MA, et al.: Content analysis of mobile health applications on diabetes mellitus. Front Endocrinol (Lausanne) 2017;8:318.

14. Greenwood DA, Gee PM, Fatkin KJ, Peeples M: A systematic review of reviews evaluating technology-enabled diabetes self-management education and support. J Diabetes Sci Technol 2017;11:1015-1027.

15. Peterson A: Improving type 1 diabetes management with mobile tools: a systematic review. J Diabetes Sci Technol 2014;8:859-864.

16. Grohmann-Izay B, Forisch M: Use of digital tracking devices in the management of diabetes mellitus: a systemic review and meta-analysis [abstract]. Diabetes 2015; 64(Suppl. 1):137-LB.

17. American Association of Diabetes Educators: Position Statement: AADE7 Self-Care Behaviors. December 2, 2014. https://www.diabeteseducator.org/docs/default-source/ legacy-docs/_resources/pdf/publications/aade7_position statement_final.pdf?sfvrsn=4. Accessed December 23, 2018. 
18. van Onzenoort HA, Menger FE, Neef C, et al.: Participation in a clinical trial enhances adherence and persistence to treatment: a retrospective cohort study. Hypertension 2011; 58:573-578.

19. Rothwell PM: External validity of randomized controlled trials: "to whom do the results of this trial apply?" Lancet 2005;365:82-93.

20. Hompesch M, Hergesheimer L, Kalcher K, et al.: Retrospective analysis of Impact on SMBG and glycemic control of mobile health (mHealth)-application for diabetes management. J Diabetes Sci Technol 2017;11:A31.

21. American Diabetes Association: eAG/A1C conversion calculator. https://professional.diabetes.org/diapro/glucose_ calc (accessed December 29, 2018).

22. Hompesch M, Kalcher K, Debong F, Morrow L: Significant improvement of blood glucose control in a high risk population of type 1 diabetes using a mobile health app: a retrospective observational study. Diabetes Technol Ther 2017;64(Suppl. 1):2337.

23. Kovatchev BP, Cox DJ, Gonder-Frederick LA, et al.: Assessment of risk for severe hypoglycemia among adults with IDDM: validation of the low blood glucose index. Diabetes Care 1998;21:1870-1875.

24. Hompesch M, Kalcher K, Debong F: High risk population using mobile logging application shows significant reduction in LBGI [abstract]. Diabetes 2017;66(Suppl. 1):952-P.

25. Hompesch M, Scheiner G, Schuster L, Kober J: Clinically relevant improvement in quality of blood glucose control in well controlled users of mySugr's mobile diabetes management tool [abstract]. Presented at the Diabetes Technology Meeting, November 8-10, 2018, Bethesda, Maryland.

26. European Medicines Agency: Guideline on clinical investigation of medicinal products in the treatment or prevention of diabetes. May 14, 2012. CPMP/EWP/1080/00 Rev. 1 Committee for Medicinal Products for Human Use (CHMP). https://www.ema.europa.eu/documents/scientific-guideline/ guideline-clinical-investigation-medicinal-products-treatmentprevention-diabetes-mellitus-revision_en.pdf. Accessed January 1, 2019.

27. Hibbits J, Koberb J, Wredeb J, et al.: Strong customer satisfaction among users of mobile diabetes management.
Presented at the Diabetes Technology Meeting. November 8-10, 2018, Bethesda, Maryland, USA.

28. Ramshaw A: NPS is 2.7 times more sensitive than Customer Satisfaction. Genroe. https://www.genroe.com/blog/netpromoter-score-is-2-7-times-more-sensitive-than-customersatisfaction/1470 (accessed January 4, 2018).

29. Reichheld F: The one number you need to grow. Harvard Business School, 2003. https://hbr.org/2003/12/the-onenumber-you-need-to-grow (accessed January 2, 2018).

30. Powers MA, Bardsley J, Cypress M, et al.: Diabetes selfmanagement education and support in type 2 diabetes: a joint position statement of the American Diabetes Association, the American Association of Diabetes Educators, and the Academy of Nutrition and Dietetics. Diabetes Educ 2015;41:417-430.

31. Diabetes Advocacy Alliance: DAA regulatory position statement. www.diabetesadvocacyalliance.org/pdf/DAA DSMT_statement_022717.pdf (accessed December 26, 2018).

32. Brzan PP, Rotman D, Pajnkihar M, Klanjsek P: Mobile applications for control and self-management of diabetes: a systematic review. J Med Syst 2016;40:210.

33. Goyal S, Marita P, Lewis GF, et al.: The systematic design of a behavioural mobile health application for the selfmanagement of type 2 diabetes. Can J Diabetes 2016;40: 95-104.

34. Khosla S, White R, Medina J, et al.: Real world evidence (RWE) - a disruptive innovation or the quiet evolution of medical evidence generation? Version 2. F1000Res 2018;7: 111.

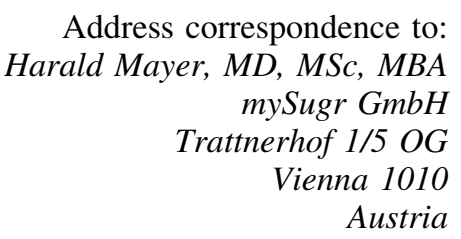

E-mail: harald.mayer@mysugr.com 